\title{
A conversation with 2013 Gairdner Wightman Award winner Dr. James Hogg
}

$\mathrm{O}$ fficer of the Order of Canada, member of the Royal Society of Canada, inductee into the Canadian Medical Hall of Fame these are among the many honours Dr. James Hogg has received for his contribution to the understanding and treatment of chronic respiratory disease. Now he can add another to the list: recipient of the Canada Gairdner Wightman Award.

Since earning an MD at the University of Manitoba in Winnipeg in 1962 and a $\mathrm{PhD}$ in experimental medicine at McGill University in Montréal, Quebec, in 1969, Hogg has clocked more than 40 years of work in pathology, pulmonary physiology and molecular biology.

His major achievement takes the form of the James Hogg Research Centre at the University of British Columbia in Vancouver. What began as a fledgling pulmonary laboratory in 1977 has grown to a 250-person strong research facility searching for solutions for heart, lung and vascular diseases.

Dr. Hogg joined $C M A J$ in a recent phone interview to discuss his life's work.

CMAJ: What sparked your interest in clinical research?

Hogg: Well, when I was a medical student, for the first time people allowed you to find something out on your own, and I liked that.

CMAJ: Why did you choose to study lung disease?

Hogg: It's a huge medical problem. It's extremely common for a start, and there are big problems it creates in terms of respiratory failure.

CMAJ: Chronic obstructive pulmonary disease has been described as a disease

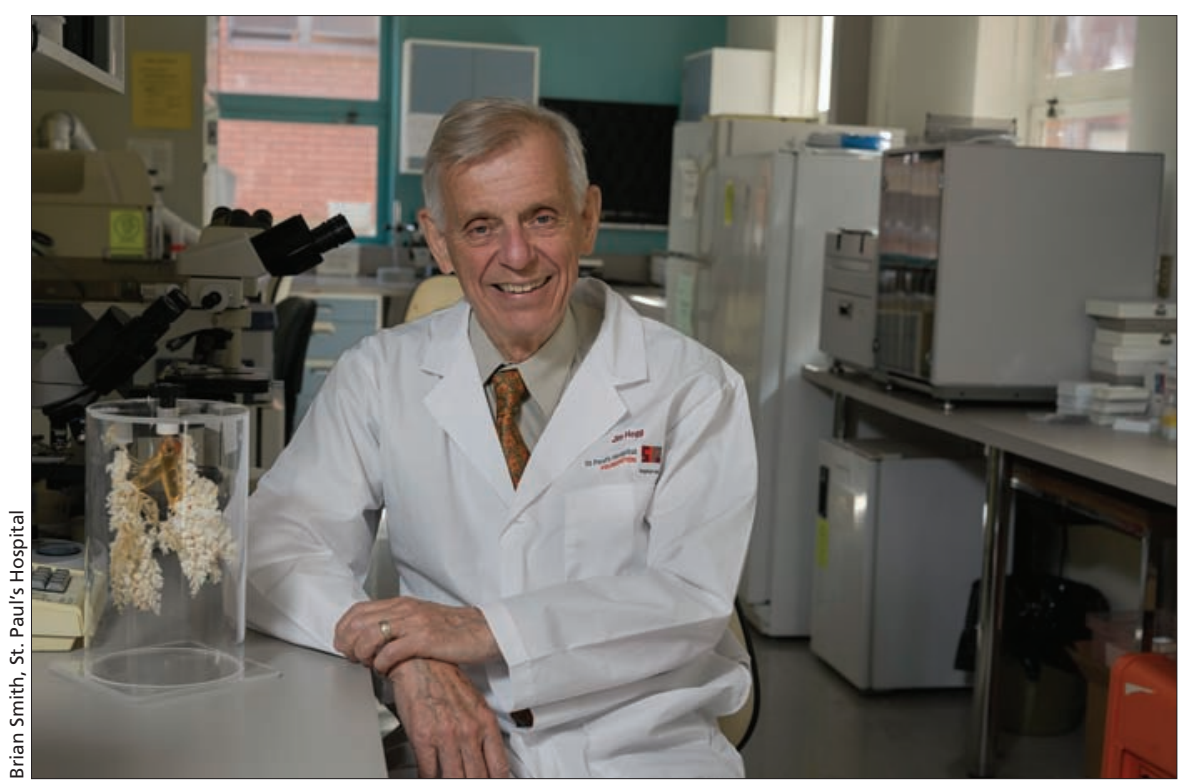

To succeed in medical research, you have to put your "heart and soul" into it, says Dr. James Hogg.

that defies easy solutions. What motivated you to sustain your focus on it for over 40 years?

Hogg: We were able to show that it's based largely on the inhalation of toxic particles, primarily from tobacco smoke. Inhalation should be treatable ... but we don't have any drugs yet to reverse the process. ... So the motivation is that it has a huge impact on people's lives, for a start, and also it has the benefit of being able to reduce the cost of medical care.

CMAJ: How did your work differ from other respiratory research happening at the time?

Hogg: We were studying lung disease by studying human lungs. Early on, we set up a tissue bank that's based on lungs from patients that had to have resection surgery for tumours and are having lung transplants. That gave us the opportunity to do some really interesting studies.
CMAJ: How does your research centre compare to the pulmonary laboratory that opened in 1977 ?

Hogg: I would say we've outstripped our initial expectations. I had no idea it would get as big as it has gotten, and so multifaceted.

CMAJ: What advice would you pass on to those entering the field today?

Hogg: To do research, you have to really want to do it. There are a lot of difficulties one way or another getting started, so you can't just do it casually. I think, more and more, you also have to have the time to do it. The competition is ferocious; you're competing against people who are working fulltime and putting their heart and soul in, so you have to have people who are willing to do that as well. - Louisa Fletcher, $C M A J$

CMAJ 2013. DOI:10.1503/cmaj.109-4654 